Supporting information

\title{
Improved and Efficient Synthesis of Chiral N,P- \\ Ligands via Cyclic Sulfamidates for Asymmetric Addition of Butyllithium to Benzaldehyde
}

Petra Rönnholm, Mikael Södergren, Göran Hilmersson

Kemivägen 10, SE-41296 Göteborg, Sweden

hilmers@chem.gu.se

Contents

1. General Experimental

S2-S3

2. Synthesis of chiral amines, experimental and spectroscopic data

S4-S9

4. $1 \mathrm{H}$ and $13 \mathrm{C}$ NMR spectra

S10-S25 


\section{Experimental}

\section{General}

NMR spectra were recorded on a Varian $400 \mathrm{MHz}$ spectrometer using $\mathrm{CDCl}_{3}$ as solvent. Optical rotations were measured using Perkin-Elmer 324 LC polarimeter. IR spectra were recorded on a Perkin-Elmer 1600 Series FTIR spectrometer. Melting points points were determined using a Büchi Melting Point B-545 and are uncorrected. GC analyses were carried out using a Varian Star 3400 CX gas chromatograph equipped with a chiral stationary phase column (CP-Chirasil-DEX $\mathrm{CB}, 25 \mathrm{~m}, 0.32 \mathrm{~mm})$ from Chrompack. Analysis were done using $\mathrm{H}_{2}(1.5 \mathrm{ml} / \mathrm{min})$ as carrier gas (injector $225^{\circ} \mathrm{C}$, detector $250^{\circ} \mathrm{C}$ ). Dried solvents were distilled from sodium/benzophenone. Column chromatography were performed with $\mathrm{SiO}_{2}-60$ (40$63 \mu \mathrm{m}$ ) from Fluka at ambient/atmospheric pressure. TLC were $\mathrm{SiO}_{2}-60 \mathrm{~F} 254$, Merck and visualized by UV light at $254 \mathrm{~nm}$ and by staining with a solution of $\mathrm{H}_{3}\left[\mathrm{P}\left(\mathrm{Mo}_{3} \mathrm{O}_{10}\right)_{4}\right] \cdot \mathrm{xH}_{2} \mathrm{O}(10 \mathrm{~g})$ in $95 \% \mathrm{EtOH}(100 \mathrm{~mL})$. MS were recorded on a Micromass LCTP using ESI+ as ionization mode and gradient of 5\% ACN to 95\% $\mathrm{ACN}$ in 6 minutes in a buffered solution of $40 \mathrm{mM}$ ammonia and $5 \mathrm{mM}$ carbonate (pH 10).

Glassware and syringes were dried at $150^{\circ} \mathrm{C}$ in a vacuum oven before transfer into a glovebox (Braun equipped with a gas purification system that removes oxygen and moisture) containing a nitrogen atmosphere. Typical moisture content was less than $1.5 \mathrm{ppm}$. Ether solvents were distilled under nitrogen from sodium/benzophenone and were kept over $4 \AA$ molecular sieves in septum sealed flasks inside the glove box.

\section{General procedure for the addition of BuLi to benzaldehyde}

$\mathrm{BuLi}(0.30 \mathrm{mmol}, 120 \mu \mathrm{L}, 2.5 \mathrm{M}$ in hexanes) was added dropwise, over a period of 5 min, to a solution of the chiral aminophosphine (0.20 mmol, 4.0 eq.) in dry $\mathrm{Et}_{2} \mathrm{O} / \mathrm{THF}$ 1:1 (2.0 mL) at $-78{ }^{\circ} \mathrm{C}$ under $\mathrm{N}_{2}$. After 15 minutes, the solution was cooled to -116 ${ }^{\circ} \mathrm{C}$, using an $\mathrm{Et}_{2} \mathrm{O} /$ liquid nitrogen cooling bath, and after a further 15 minutes at this temperature, a solution of benzaldehyde (1.0 $\mathrm{M}$ in hexane, containing n-decane as an 
internal standard, $50 \mu \mathrm{L}, 0.05 \mathrm{mmol}, 1.0$ eq.) was added dropwise, during ca $5 \mathrm{~min}$. The mixture was allowed to react for another 15 minutes before methanol (1.0 mL) was added to quench the remaining organolithum species. The resulting mixture was allowed to warm up to room temperature and aqueous $\mathrm{HCl}(1 \mathrm{M}, 1.0 \mathrm{~mL})$ and MTBE $(2.0 \mathrm{~mL})$ were added, followed by rigorous stirring during $5 \mathrm{~min}$. The stirring was discontinued and an aliquot of the organic phase was diluted with MTBE and analysed using chiral stationary phase gas chromatography. 


\section{Syntheses of the chiral amines}

\section{(4R)-3-isopropyl-4-phenyl-1,2,3-oxothiazolidine $S$-oxide (4c)}

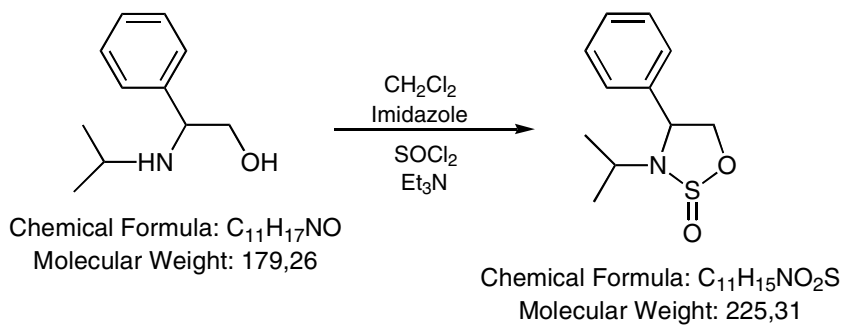

A solution of (R)- $N$-isopropyl-phenylglycinol (47.0 g, $0.26 \mathrm{~mol}$ ), imidazole (72 g, 1.1 $\mathrm{mmol})$, and $\mathrm{Et}_{3} \mathrm{~N}(73 \mathrm{~mL}, 0.52 \mathrm{~mol})$ in dry $\mathrm{CH}_{2} \mathrm{Cl}_{2}(800 \mathrm{~mL})$ was cooled on an ice bath. To the stirred yellow solution, $\mathrm{SOCl}_{2}(28 \mathrm{~mL}, 0.39 \mathrm{~mol})$ was added dropwise over ca $20 \mathrm{~min}$, keeping the internal temperature below $+10{ }^{\circ} \mathrm{C}$. After complete addition the ice bath was removed and the resulting mixture allowed to warm to rt overnight, and then filtrated through a short pad of silica gel, washed with brine, dried over $\mathrm{MgSO}_{4}$, and concentrated to give $4 \mathrm{c}$ (58.0 g, 98\% crude) as a yellowish oil, which solidified upon standing. The crude product, which was used in the next step without further purification, was judged $>95 \%$ pure by ${ }^{1} \mathrm{H}$ NMR and GC. If needed, the mixture of sulfamidites can be purified, but not easily separated into the individual diastereomers, using silica gel chromatography (silica, hexane / EtOAc 9:1 to 3:1). Filtration and concentration in vacuo gave the crude product as a brown solid. NMR of the crude product shows the desired sulfamidites as a mixture of two diastereoisomers.

${ }^{1} \mathrm{H}$ NMR $\left.\left(\mathrm{CDCl}_{3}\right): \delta 1.16(\mathrm{~d} J=6.6 \mathrm{~Hz}, 6 \mathrm{H}), 1.34(\mathrm{~d}, J=6.8 \mathrm{~Hz}, 3 \mathrm{H}),\right), 1.39(\mathrm{~d}, J=$ $6.8 \mathrm{~Hz}, 3 \mathrm{H}), 3.3-3.4(\mathrm{~m}, 1 \mathrm{H}), 4.2$ (dd, J=3.8 Hz, 1H), 4.5-4.6 (m, 2H), 4.74 (dd, $J=3.8 \mathrm{~Hz}, 1 \mathrm{H}), 4.86(\mathrm{dd}, J=6.8 \mathrm{~Hz}, 1 \mathrm{H}), 5.07$ (dd, $J=7.8 \mathrm{~Hz}, 1 \mathrm{H}), 7.3-7.6$ (m, 5H). ${ }^{13} \mathrm{C} \mathrm{NMR}\left(\mathrm{CDCl}_{3}\right): \delta 21.0,21.9,22.1,23.0,46.7,48.2,60.0,65.9,76.0,127.9,128.1$, $128.5,129.1,137.1,138.0$.

\section{(4S)-3-isopropyl-4-isopropyl-1,2,3-oxothiazolidine $S$-oxide (4a)}

Using the procedure described for $4 \mathbf{c},(S)$ - $N$-isopropylvalinol (28 g, $0.20 \mathrm{~mol}$ ) gave 4a as a 1:1 mixture of inseparable diastereoisomers (36.5 g, 97\%), as a yellow oil, which solidified upon standing. The crude product thus obtained was directly used in the next step without further purification. 
${ }^{1} \mathrm{H}$ NMR $\left(\mathrm{CDCl}_{3}\right): \delta 0.82(\mathrm{~d}, J=6.9 \mathrm{~Hz}, 3 \mathrm{H}), 0.92(\mathrm{~d}, J=7.0 \mathrm{~Hz}, 3 \mathrm{H}), 0.98(\mathrm{~d}, J=6.8$ $\mathrm{Hz}, 3 \mathrm{H}), 1.20$ (d, J=6.9 Hz, 3H), 1.21 (d, J=6.6 Hz, 3H), 1.3 (d, J=6.6 Hz, 3H), 1.36 (d, J=6.9 Hz, 3H), 1.47 (d, J=6.9 Hz, 3H), 3.25 (q, $J=7.57 \mathrm{~Hz}, 1 \mathrm{H}), 3.38$ (q, $J=6.70$ $\mathrm{Hz}, 1 \mathrm{H}), 3.52$ (q, J=6.70 Hz, 1H), 3.68-3.74 (m, 1H), 4.24 (dd, J=8.80 Hz, 1H), 4.49 (dd, $J=7.37 \mathrm{~Hz}, 1 \mathrm{H}), 4.57$ (dd, $J=8.42 \mathrm{~Hz}, 1 \mathrm{H}), 4.70$ (dd, $J=7.74 \mathrm{~Hz}, 1 \mathrm{H})$.

${ }^{13} \mathrm{C} \mathrm{NMR}\left(\mathrm{CDCl}_{3}\right): \delta 15.02,18.37,19.62,20.40,21.07,21.43,22.26,23.11,28.64$, $31.95,46.86,50.85,60.41,67.26,69.60,72.34$.

\section{(4S)-3-isopropyl-4-benzyl-1,2,3-oxothiazolidine $S$-oxide (4b)}

Using the procedure described for 4c, $(S)$ - $N$-isopropyl-phenylalaninol (6.5 g, 34 mmol) gave 4b s a 1:1 mixture of inseparable diastereoisomers (7.5 g, 87\%), as a brown oil, which solidified upon standing. The crude product thus obtained was directly used in the next step without further purification.

(S)-phenylalanine cyclic sulfamidite, mixture of 1:1 epimers at sulfur: ${ }^{1} \mathrm{H} \mathrm{NMR}\left(\mathrm{CDCl}_{3}\right): \delta 1.21(\mathrm{~d}, J=6.7 \mathrm{~Hz}, 3 \mathrm{H}), 1.35(\mathrm{~d}, J=6.7 \mathrm{~Hz}, 6 \mathrm{H}), 1.35(\mathrm{~d}, J=$ $6.7 \mathrm{~Hz}, 3 \mathrm{H}), 2.56(\mathrm{dd}, J=9.8,13.5 \mathrm{~Hz}, 1 \mathrm{H}), 2.91-3.04(\mathrm{~m}, 2 \mathrm{H}), 3.23(\mathrm{dd}, J=5.8$, $13.5 \mathrm{~Hz}, 1 \mathrm{H}$ ), 3.46 (sept, $J=6.7 \mathrm{~Hz}, 1 \mathrm{H}$ ), 3.61 (sept, $J=6.7 \mathrm{~Hz}, 1 \mathrm{H}$ ), 3.69-3.79 (m, $1 \mathrm{H}), 3.93-4.02(\mathrm{~m}, 1 \mathrm{H}), 4.16(\mathrm{t}, J=4.0,8.6 \mathrm{~Hz}, 1 \mathrm{H}), 4.29-4.37(\mathrm{~m}, 1 \mathrm{H}), 4.54-4.66$ $(\mathrm{m}, 2 \mathrm{H})$.

${ }^{13} \mathrm{C} \mathrm{NMR}\left(\mathrm{CDCl}_{3}\right): \delta 21.5,22.0,22.20,22.7,39.5,40.7,47.0,48.5,57.4,61.5,72.9$, 75.6, 126.9, 127.1, 128.9, 129.2, 137.2, 138.0.

\section{(4R)-3-isopropyl-4-phenyl-1,2,3-oxothiazolidine $S, S$-dioxide (5c)}

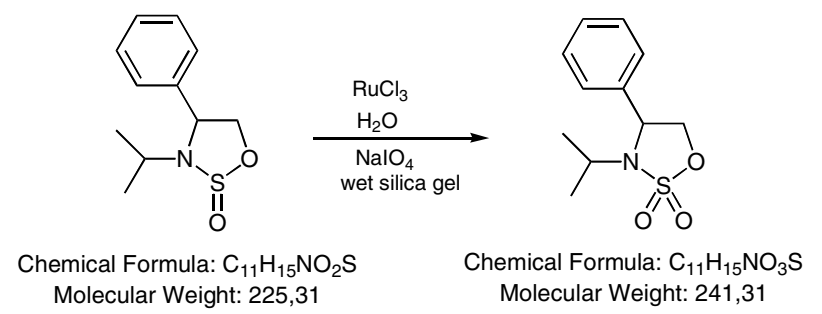

$\mathrm{RuCl}_{3}(0.7 \mathrm{mg}, 0.15 \mathrm{~mol} \%)$ was added to water $(1.25 \mathrm{ml})$ yielding a black solution. This solution was stirred at a brisk pace and small portions of $\mathrm{NaIO}_{4}(1.19 \mathrm{~g}, 5.55$ mmol) were then added, the black solution immediately turned yellow $\left(\mathrm{RuO}_{4}\right)$. Note that only the amount of $\mathrm{NaIO}_{4}$ that dissolved was added. The $\mathrm{RuO}_{4}$ solution and the remaining $\mathrm{NaIO}_{4}$ was added directly to silica gel $(2.5 \mathrm{~g})$ in a $100 \mathrm{ml}$ round bottom flask with a magnetic stirring bar. The rate of stirring was increased and the solid was 
stirred until a free flowing white solid was obtained. EtOAc $(9 \mathrm{ml})$ was added to the wet silica gel and put on an ice bath. The sulfamidite (500 mg, $2.22 \mathrm{mmol}$ ) was dissolved in EtOAc $(9 \mathrm{ml})$, a few drops of $\mathrm{CH}_{2} \mathrm{Cl}_{2}$ was added to dissolve all of the sulfamidite. The solution of the substrate was added dropwise to the slurry of silica gel at $0{ }^{\circ} \mathrm{C}$. After complete addition the ice bath was removed and stirred at a brisk pace throughout the reaction. A white slurry is formed and stirred until TLC (silica, hexane/EtOAc 3:1) indicated complete consumption of the starting material, Typically this requires $10 \mathrm{~min}-2 \mathrm{~h}$ depending on sulfamidite. The slurry was filtrated through a short pad of silica gel, dried over $\mathrm{Na}_{2} \mathrm{SO}_{4}$, filtrated and concentrated in vacuo to give $\mathbf{5 c}(503.3 \mathrm{mg}, 2.086, \mathrm{mmol} 95 \%$ crude) as a colourless oil as crude product, which solidifies upon standing. In most cases, the crude product was essentially pure ( $>95 \%$ by NMR and GC) and used in the next step without further purification. Occasionally, however, the crude product contains traces of the $\mathrm{Ru}$ catalyst (brown to black). This may be removed by re-dissolving in hexane/EtOAc (4:1) and filtering again through a silica plug (ca $5 \mathrm{~cm}$ ), followed by evaporation.

${ }^{1} \mathrm{H}$ NMR $\left(\mathrm{CDCl}_{3}\right): \delta 1.13(\mathrm{~d}, J=6.73 \mathrm{~Hz} 3 \mathrm{H}), 1.37(\mathrm{~d}, J=6.89 \mathrm{~Hz} 3 \mathrm{H}), 3.7-3.6(\mathrm{~m}, 1 \mathrm{H})$, 4.24-4.30 (dd, $J=8.17,8.17 \mathrm{~Hz} 1 \mathrm{H}$ ), 4.64-4.70 (dd, $J=15.52,15.52 \mathrm{~Hz}, 1 \mathrm{H}), 4.80-4.86$ (dd, $J=7.33,7.33 \mathrm{~Hz}, 1 \mathrm{H}), 7.39-7.47$ (m, 5H).

${ }^{13} \mathrm{C} \mathrm{NMR}\left(\mathrm{CDCl}_{3}\right): \delta 19.82,20.12,49.09,60.73,72.49,127.40,129.44,129.51$, 136.90 .

$v_{\max }$ (neat): 2981, 2359, 1604, 1456, 1338, $1194 \mathrm{~cm}^{-1}$.

$[\alpha]_{D}^{20}=-87.3^{\circ}\left(\mathrm{c}=1.00, \mathrm{CDCl}_{3}\right)$.

HRMS (FAB): $\mathrm{MH}^{+}$, found 242.0856, $\mathrm{C}_{11} \mathrm{H}_{16} \mathrm{NO}_{3} \mathrm{~S}$ requires 242.0853.

\section{(4S)-3-isopropyl-4-isopropyl-1,2,3-oxothiazolidine $S, S$-dioxide (5a)}

Following the procedure described for $\mathbf{5 c}$, sulfamidate $\mathbf{4 a}(2.00 \mathrm{~g}, 10.5 \mathrm{mmol})$ was oxidized to give $\mathbf{5 a}(2.02 \mathrm{~g}, 93 \%)$ as a colourless oil.

${ }^{1} \mathrm{H}$ NMR $\left(\mathrm{CDCl}_{3}\right): \delta 0.97(\mathrm{dd}, J=7.38 \mathrm{~Hz}, 6 \mathrm{H}), 1.29(\mathrm{~d}, J=6.83 \mathrm{~Hz}, 3 \mathrm{H}), 1.36(\mathrm{~d}$, $J=6.83 \mathrm{~Hz}, 3 \mathrm{H}), 2.03$ (sext, 1H), 3.50 (sext, 1H), 3.70 (q, 1H), 4.25 (dd, $J=8.82 \mathrm{~Hz}$, $1 \mathrm{H}), 4.40(\mathrm{~m}, 1 \mathrm{H})$.

${ }^{13} \mathrm{C} \mathrm{NMR}\left(\mathrm{CDCl}_{3}\right): \delta 16.49,18.89,19.64,20.57,30.87,50.63,61.44,67.93$.

$v_{\max }$ (neat): $2969,2359,1718,1469,1338,1196 \mathrm{~cm}^{-1}$.

$[\alpha]_{D}^{20}=-12.11\left(\mathrm{c}=1.14, \mathrm{CDCl}_{3}\right)$. 
HRMS (FAB): $\mathrm{MH}^{+}$, found 208.1011, $\mathrm{C}_{8} \mathrm{H}_{18} \mathrm{NO}_{3} \mathrm{~S}$ requires 208.1009.

\section{(4S)-3-isopropyl-4-benzyl-1,2,3-oxothiazolidine $S, S$-dioxide (5b)}

Following the procedure described for 5c, sulfamidate $\mathbf{4 b}(5.49 \mathrm{~g}, 28.76 \mathrm{mmol})$ was oxidized to give $\mathbf{5 b}(6.39 \mathrm{~g}, 93 \%)$ as a colourless oil.

${ }^{1} \mathrm{H}$ NMR $\left(\mathrm{CDCl}_{3}\right): \delta 1.11(\mathrm{~d}, J=6.79 \mathrm{~Hz} 3 \mathrm{H}), 1.32$ (d, $\left.J=6.82 \mathrm{~Hz} 3 \mathrm{H}\right), 2.85-2.95(\mathrm{~m}$, $1 \mathrm{H}), 3.10-3.20$ (m, 1H), 3.68 (sext, $J=6.8 \mathrm{~Hz}, 1 \mathrm{H}), 3.90-4.00$ (m, 1H), 4.20-4.30 (m, $1 \mathrm{H}), 4.20-4.35(\mathrm{~m}, 1 \mathrm{H}), 7.20-7.45(\mathrm{~m}, 5 \mathrm{H})$.

${ }^{13} \mathrm{C}$ NMR $\left(\mathrm{CDCl}_{3}\right): 19.28,20.66,39.75,50.13,57.36,70.68,127.20,128.78,129.38$, 135.90

$V_{\max }\left(\right.$ neat): $2979,2257,1604,1455,1338,1189 \mathrm{~cm}^{-1}$.

\section{(R)-2-isopropylamino-2-phenyl-1-(diphenylphosphino)ethane (6c)}

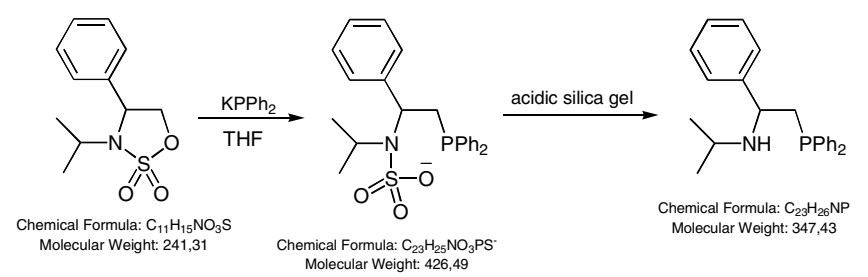

To a solution of $5 \mathbf{c}(1.0 \mathrm{~g}, 4.14 \mathrm{mmol})$ in dry THF $(5 \mathrm{~mL})$ at $-78{ }^{\circ} \mathrm{C}$, a solution of $\mathrm{KPPh}_{2}(0.5 \mathrm{M}$ in THF, $8.7 \mathrm{~mL}, 4.35 \mathrm{mmol})$ was added dropwise, over $15 \mathrm{~min}$, to give a red solution. After complete additon the ice bath was removed and stirred for another $5 \mathrm{~min}$, when TLC (hexane/EtOAc/Et $3 \mathrm{~N}, 10: 1: 0.1$ ) indicated complete disappearance of the starting material (5-45 min depending on the sulfamidate and concentration in the reaction mixture). Acidic silica $(2 \mathrm{~g}$ silica and $0.8 \mathrm{~mL} 2 \mathrm{M}$ aq. $\mathrm{H}_{2} \mathrm{SO}_{4}$, evenly mixed to a white powder) was added portionwise until $\mathrm{pH} 1$. The resulting white slurry was stirred at rt during $40 \mathrm{~min}$, followed by the addition of saturated aqueous $\mathrm{NaHCO}_{3}(35 \mathrm{~mL})$. The mixture was filtered off and washed with brine $(2 \times 10 \mathrm{~mL})$. The combined aqueous layers were extracted with $\mathrm{CH}_{2} \mathrm{Cl}_{2}(3 \times 10$ $\mathrm{mL}$ ) and the combined organic layers were dried over $\mathrm{Na}_{2} \mathrm{SO}_{4}$ and evaporated under reduced pressure to give a white solid (1.75 g, 122\% crude), which was purified by column chromatography (hexane/EtOAc/Et $3 \mathrm{~N}, 10: 1: 0.1)$ to yield $\mathbf{6 c}(1.17 \mathrm{~g}, 81 \%)$ as a colourless oil, which solidified upon standing.

${ }^{1} \mathrm{H} \mathrm{NMR}\left(\mathrm{CDCl}_{3}\right): \delta 0.94(\mathrm{t}, J=5.52 \mathrm{~Hz}, 6 \mathrm{H}), 1.6(\mathrm{bs}, 1 \mathrm{H}), 2.44(\mathrm{~d}, 2 \mathrm{H}, J=6.93 \mathrm{~Hz})$, 2.50-2.60 (m, 1H), 3.75-3.80 (q, $J=7.13 \mathrm{~Hz}, 1 \mathrm{H}), 7.20-7.50(\mathrm{~m}, 15 \mathrm{H})$. 
${ }^{13} \mathrm{C} \mathrm{NMR}\left(\mathrm{CDCl}_{3}\right): \delta 22.0,24.26,38.99,39.13,45.87,57.44,57.59,126.80,126.98$, $128.31,128.38,128.75,132.47,132.66,133.06,133.25,138.05,138.18,138.77$, 138.89, 145.15, 145.21 .

${ }^{31} \mathrm{P}$ NMR $\left(\mathrm{CDCl}_{3}\right): \delta-22.30$ $v_{\max }$ (neat): 3314, 3055, 2960, 1952, 1883, 1811, 1585, 1477, 1433, 1378, $1169 \mathrm{~cm}^{-1}$. $[\alpha]_{D}^{20}=-60.12^{\circ}(\mathrm{c}=1.60, \mathrm{THF})$.

HRMS (FAB): $\mathrm{MH}^{+}$, found 348.1883, $\mathrm{C}_{23} \mathrm{H}_{27} \mathrm{NP}$ requires 348.1912.

\section{(S)-2-isopropylamino-3-metyl-1-(diphenylphosphino)butane (6a)}

Using the procedure described for the synthesis of $\mathbf{6 b}$, starting from $\mathbf{5 a}(0.79 \mathrm{~g}, 3.83$ mmol) was phosphinated to yield $6 \mathbf{a}(0.55 \mathrm{~g}, 46 \%$ yield $)$ as a colourless syrup.

${ }^{1} \mathrm{H} \mathrm{NMR}\left(\mathrm{CDCl}_{3}\right): \delta 0.83(\mathrm{~d}, J=6.86 \mathrm{~Hz}, 3 \mathrm{H}, 0.89(\mathrm{~d}, J=6.86 \mathrm{~Hz}, 3 \mathrm{H}), 0.93(\mathrm{t}, J$ $=6.86 \mathrm{~Hz}, 6 \mathrm{H}), 1.94-2.05(\mathrm{~m}, 2 \mathrm{H}), 2.23(\mathrm{~m}, 1 \mathrm{H}), 2.48(\mathrm{~m}, 1 \mathrm{H}), J=4.26 \mathrm{~Hz}), 2.77(\mathrm{~m}$, $1 \mathrm{H}), 7.35-7.50(\mathrm{~m}, 10 \mathrm{H})$.

${ }^{13} \mathrm{C} \mathrm{NMR}\left(\mathrm{CDCl}_{3}\right): \delta 17.20,18.13,23.11,23.73,30.52,30.60,30.86,30.99,45.96$, $56.85,56.97,128.22$, 128.29, 128.35, 128.63, 132.43, 132.61, 133.06, 133.25, 138.45, $138.48,139.50,139.63$.

${ }^{31} \mathrm{P} \mathrm{NMR}\left(\mathrm{CDCl}_{3}\right): \delta-21.68$ $v_{\max }$ (neat): 3314, 3053, 2957, 1952, 1883, 1810, 1585, 1477, 1434, 1377, $1169 \mathrm{~cm}^{-1}$. $[\alpha]_{D}^{20}=+37.04^{\circ}(\mathrm{c}=1.73, \mathrm{THF})$.

HRMS (FAB): $\mathrm{MH}^{+}$, found 314.2015, $\mathrm{C}_{20} \mathrm{H}_{29} \mathrm{NP}$ requires 314.2039.

\section{(S)-2-isopropylamino-3-phenyl-1-(diphenylphosphino)propane (6b)}

Using the procedure described for the phosphination of $\mathbf{6 c}$, sulfamidate $\mathbf{5 b}(0.79 \mathrm{~g}$, $3.83 \mathrm{mmol})$ was converted into $\mathbf{6 b}(1.10 \mathrm{~g}, 4.3 \mathrm{mmol}, 80 \%$ yield $)$ as a viscous, colourless oil.

${ }^{1} \mathrm{H} \mathrm{NMR}\left(\mathrm{CDCl}_{3}\right): \delta 0.86(\mathrm{~d}, J=6.27 \mathrm{~Hz}, 3 \mathrm{H}), 0.91(\mathrm{~d}, J=6.27 \mathrm{~Hz}, 3 \mathrm{H}), 1.1-1.2(\mathrm{bs}$, $1 \mathrm{H}), 2.11(\mathrm{t}, J=6.6 \mathrm{~Hz}, 1 \mathrm{H}), 2.23(\mathrm{~m}, 1 \mathrm{H}), 2.76-2.92(\mathrm{~m}, 4 \mathrm{H})$.

${ }^{13} \mathrm{C}$ NMR $\left(\mathrm{CDCl}_{3}\right): \delta 22.76,23.97,33.58,34.29,42.30,42.40,45.94,52.19,52.22$, $126.54,128.80,129.78,130.65,130.75,130.95,131.04,131.78,132.50,133.47$, $133.70,134.68,138.79$.

${ }^{31} \mathrm{P} \mathrm{NMR}\left(\mathrm{CDCl}_{3}\right): \delta-22.97$ 
$v_{\max }$ (neat): 3314, 3056, 2960, 1951, 1882, 1810, 1585, 1478, 1434, 1377, $1171 \mathrm{~cm}^{-1}$. $[\alpha]_{D}^{20}=+25.74^{\circ}(\mathrm{c}=2.02$, THF $)$.

HRMS (FAB): $\mathrm{MH}^{+}$, found 362.2043, $\mathrm{C}_{24} \mathrm{H}_{29} \mathrm{NP}$ requires 362.2039. 
${ }^{1} \mathrm{H}$ NMR (4R)-3-isopropyl-4-phenyl-1,2,3-oxothiazolidine $S$-oxide (4c)

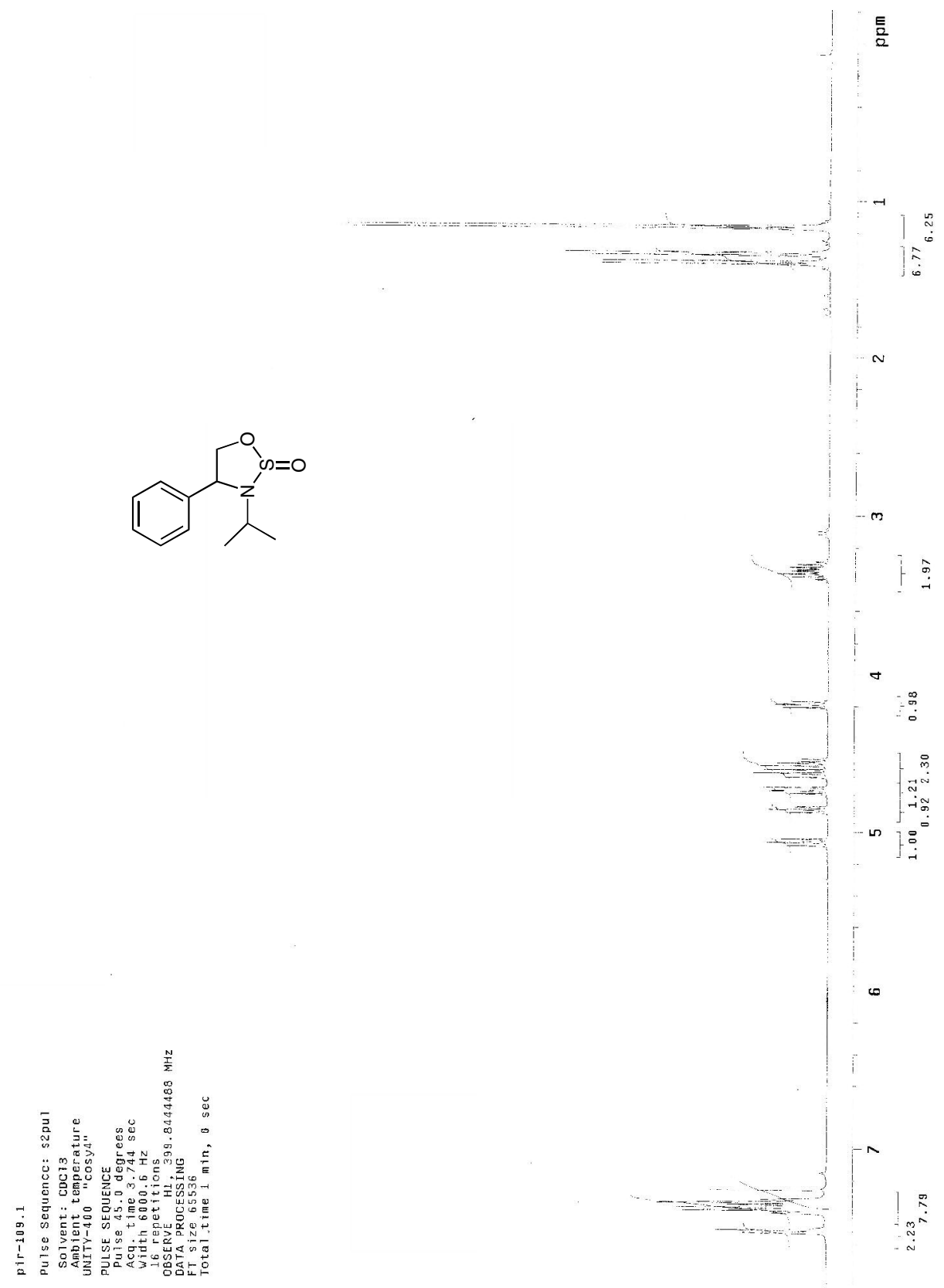


${ }^{13}$ C NMR (4R)-3-isopropyl-4-phenyl-1,2,3-oxothiazolidine $S$-oxide (4c)

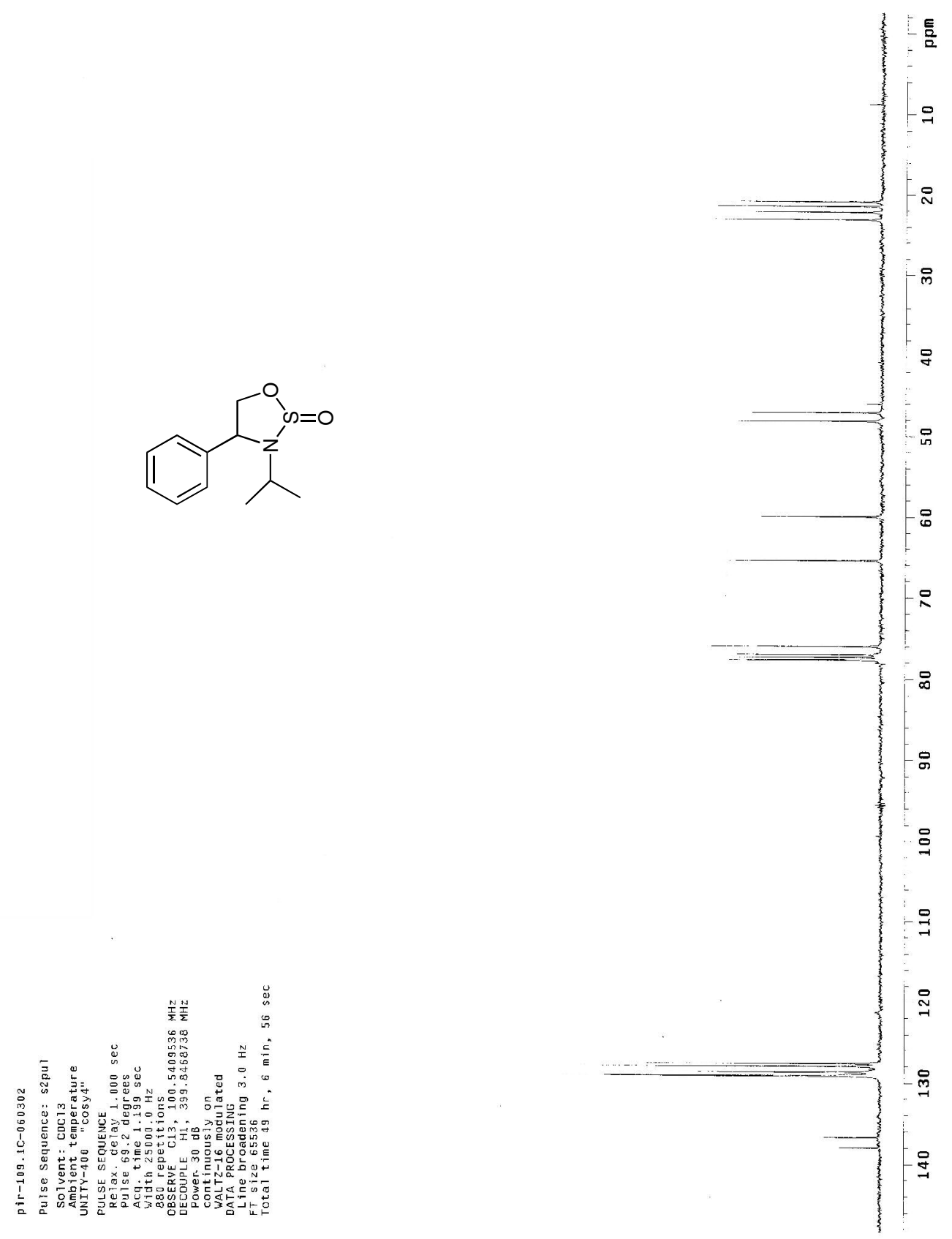


${ }^{1}$ H NMR (4S)-3-isopropyl-4-isopropyl-1,2,3-oxothiazolidine $S$-oxide (4a)

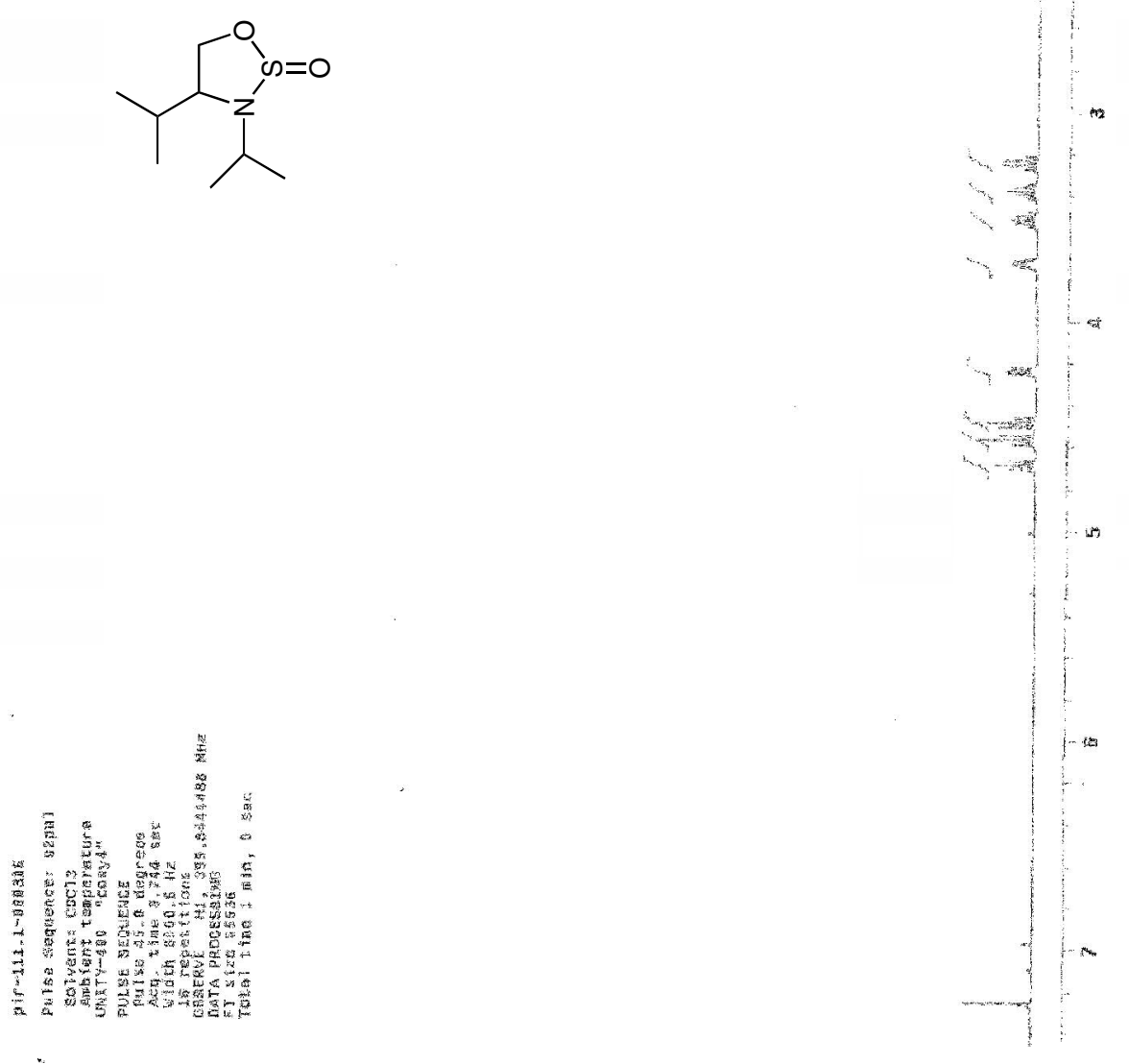


${ }^{13}$ C NMR (4S)-3-isopropyl-4-isopropyl-1,2,3-oxothiazolidine $S$-oxide (4a)

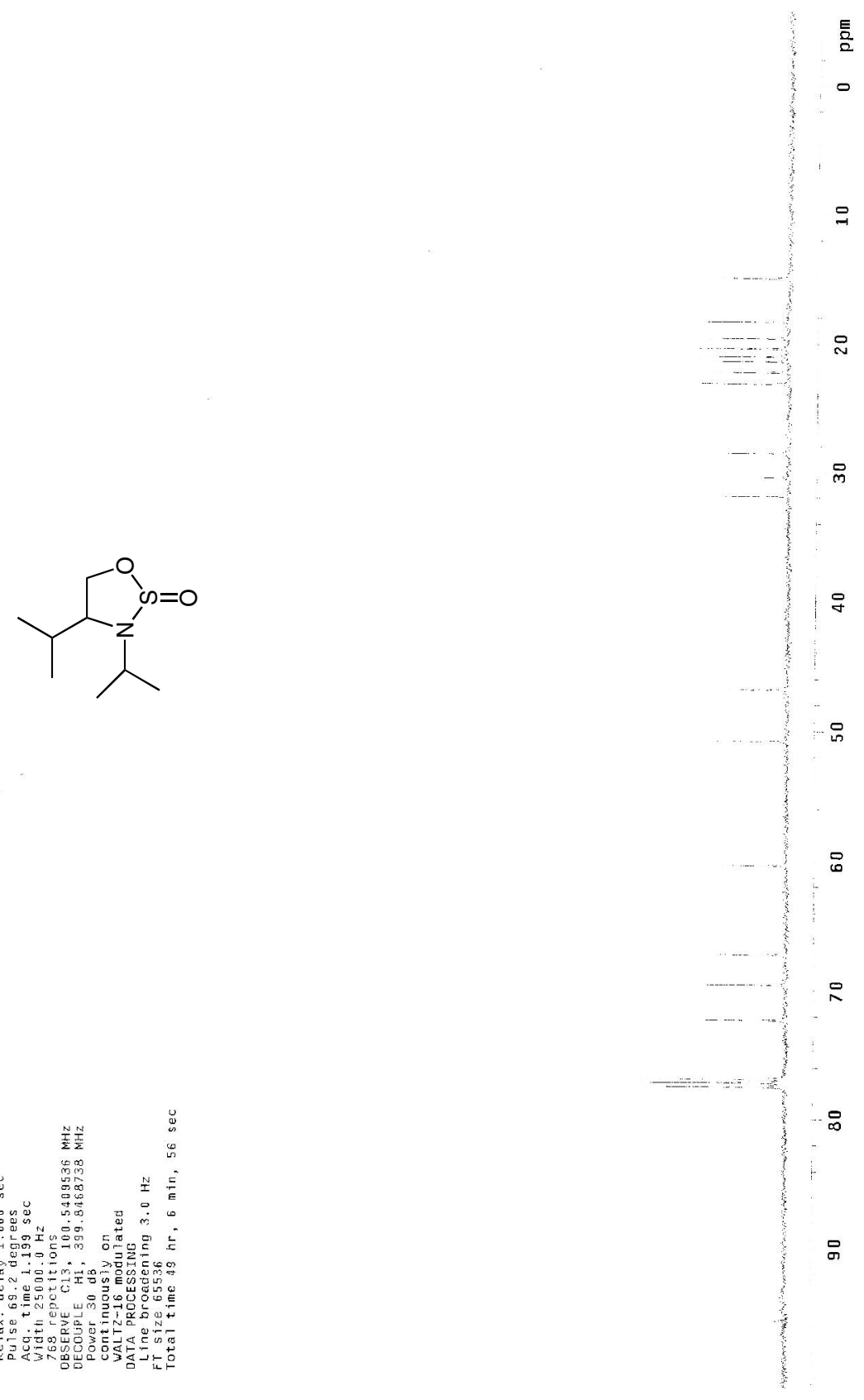


${ }^{1} \mathrm{H}$ NMR (4S)-3-isopropyl-4-phenyl-1,2,3-oxothiazolidine $S, S$-dioxide (5c)

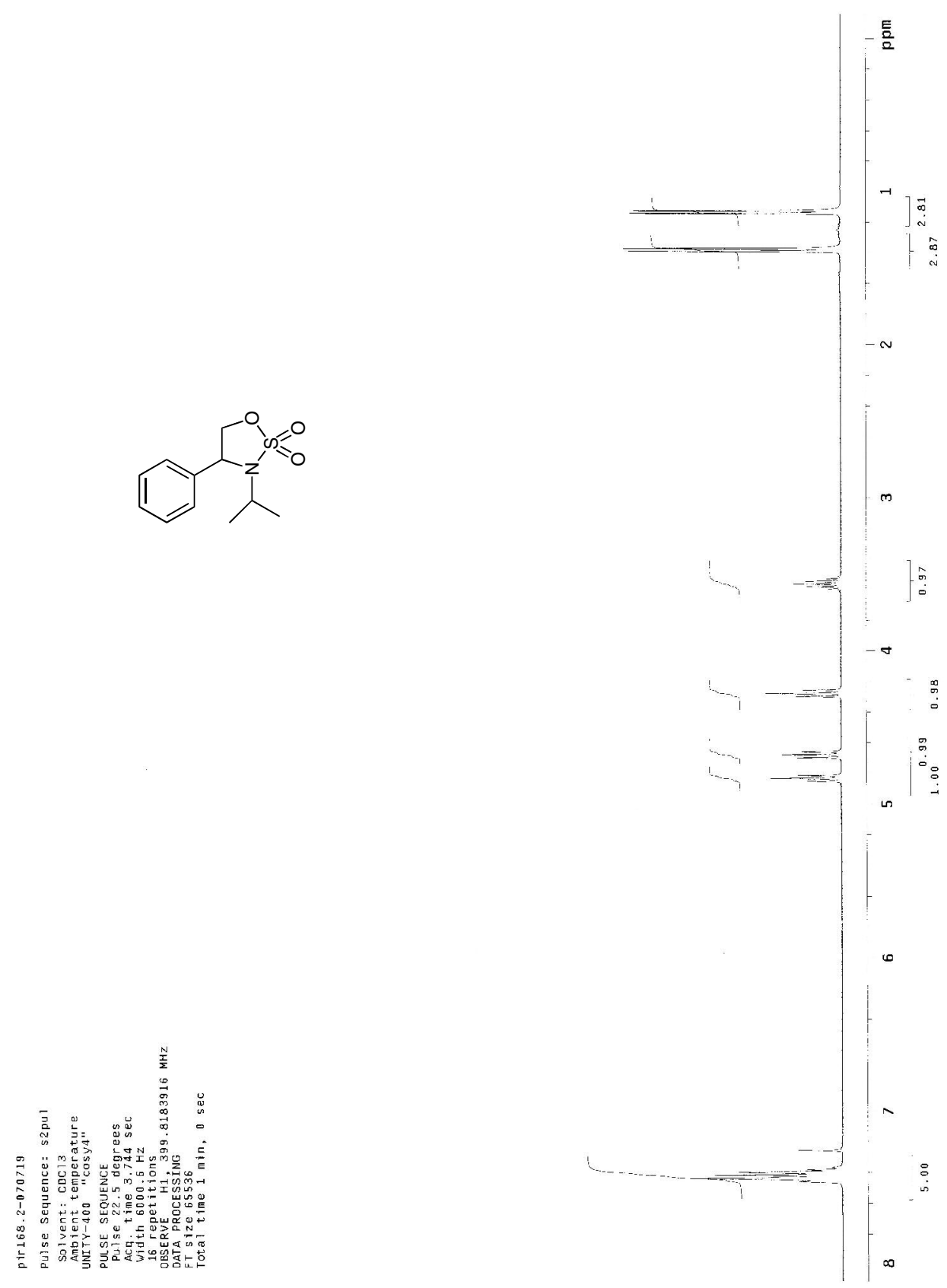


${ }^{13} \mathrm{C}$ NMR (4S)-3-isopropyl-4-phenyl-1,2,3-oxothiazolidine $S, S$-dioxide (5c)

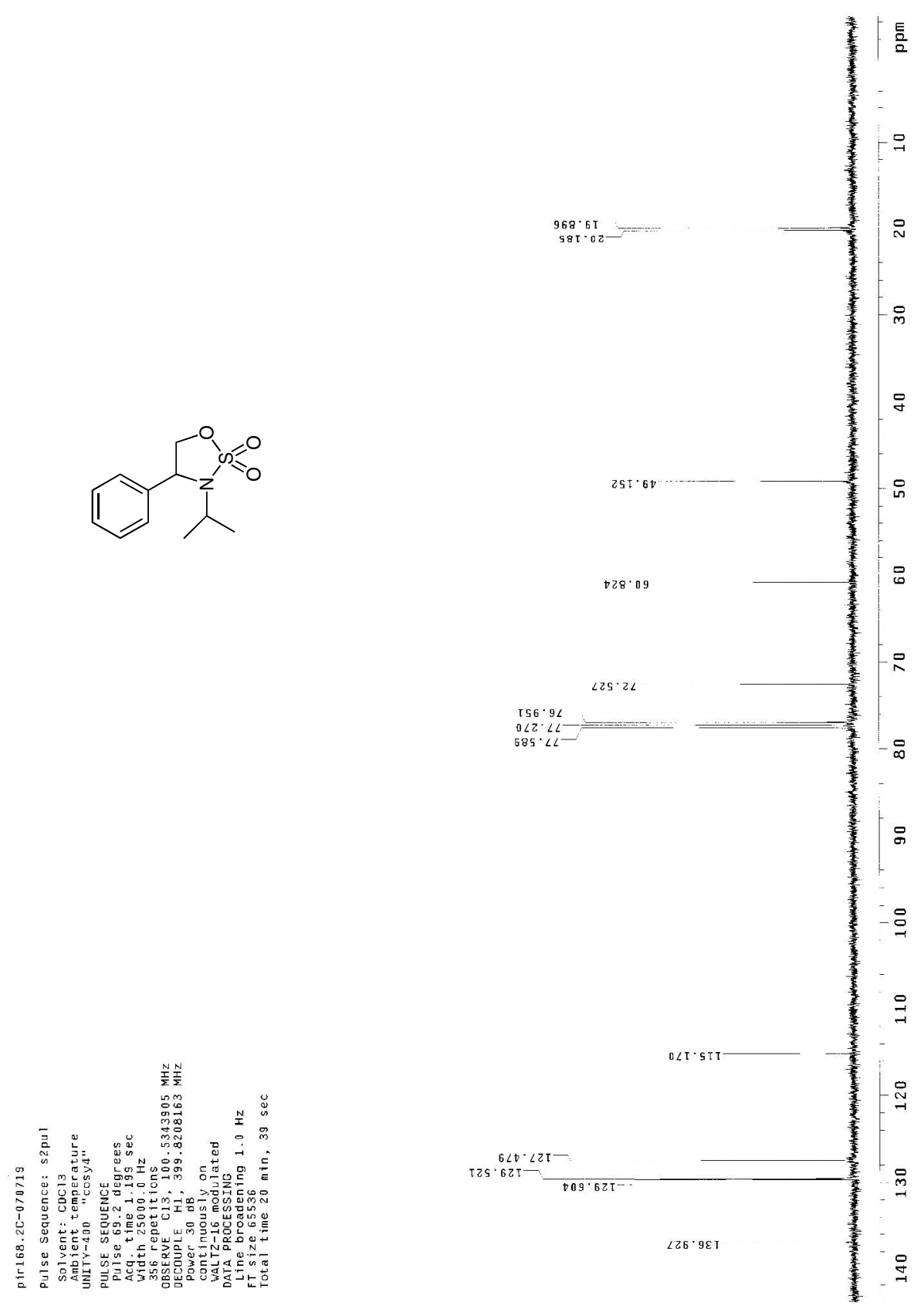


${ }^{1} \mathrm{H}$ NMR (4S)-3-isopropyl-4-isopropyl-1,2,3-oxothiazolidine $S, S$-dioxide (5a)

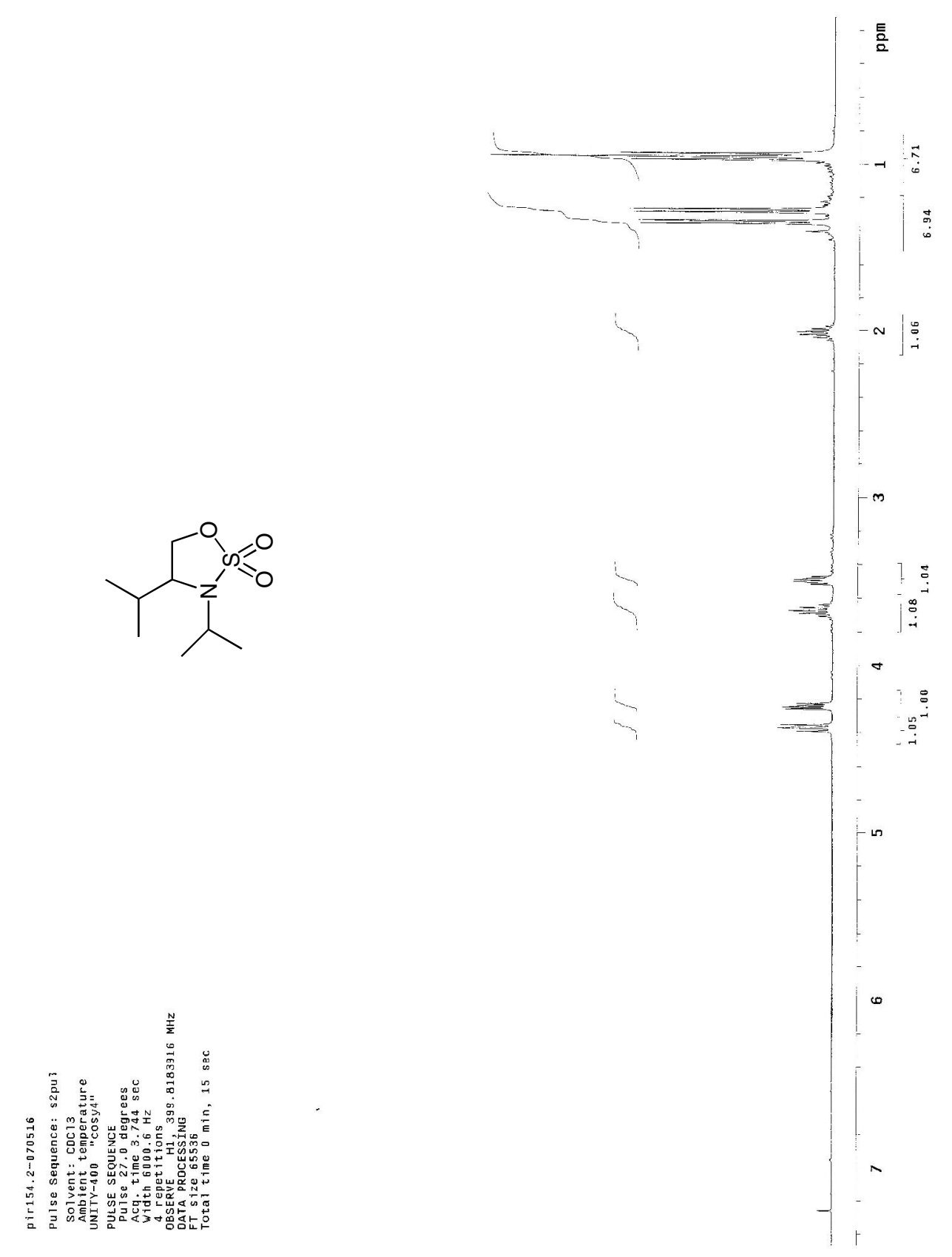


${ }^{13} \mathrm{C}$ NMR (4S)-3-isopropyl-4-isopropyl-1,2,3-oxothiazolidine $S, S$-dioxide (5a)

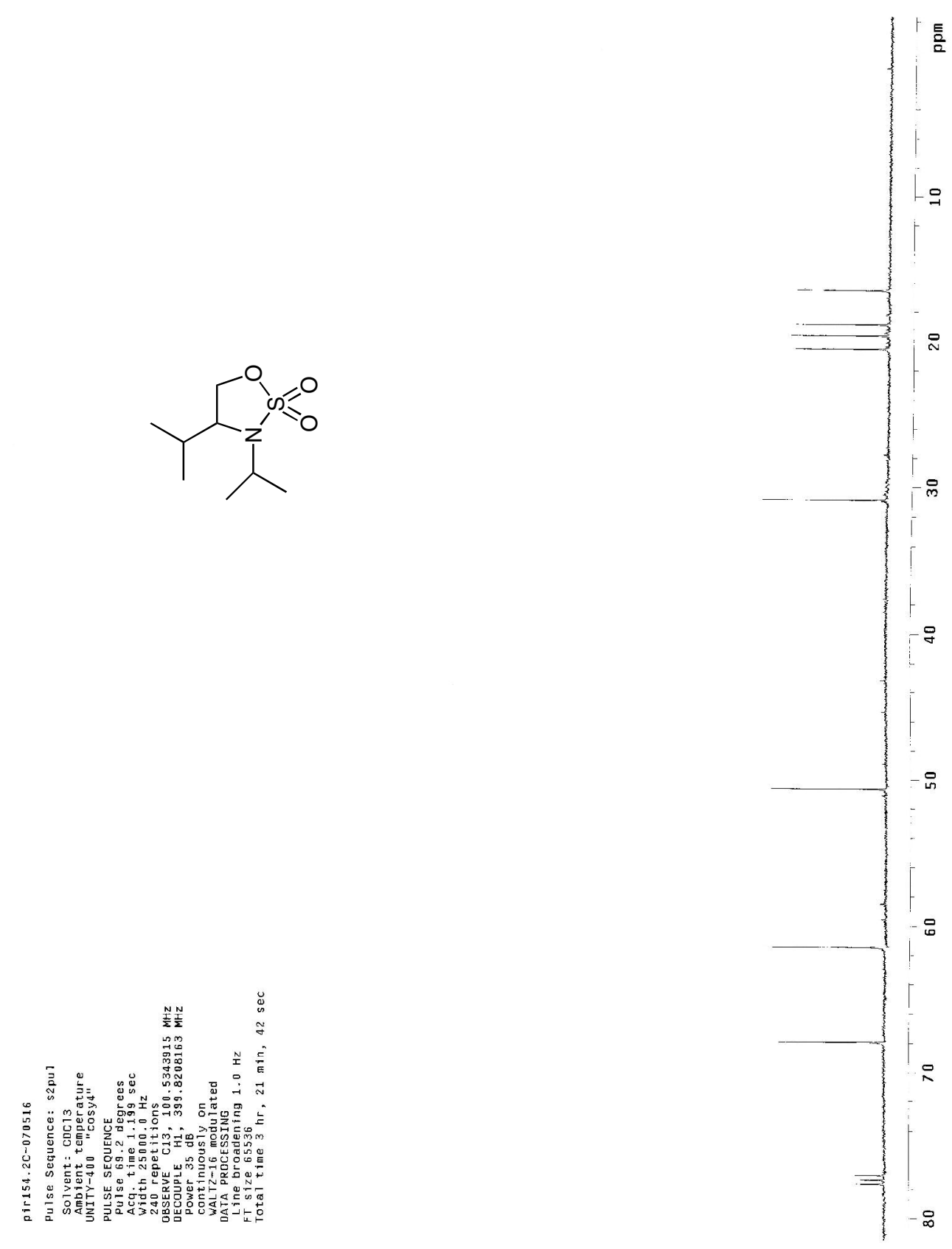


${ }^{1}$ H NMR (4S)-3-isopropyl-4-benzyl-1,2,3-oxothiazolidine $S, S$-dioxide (5b)

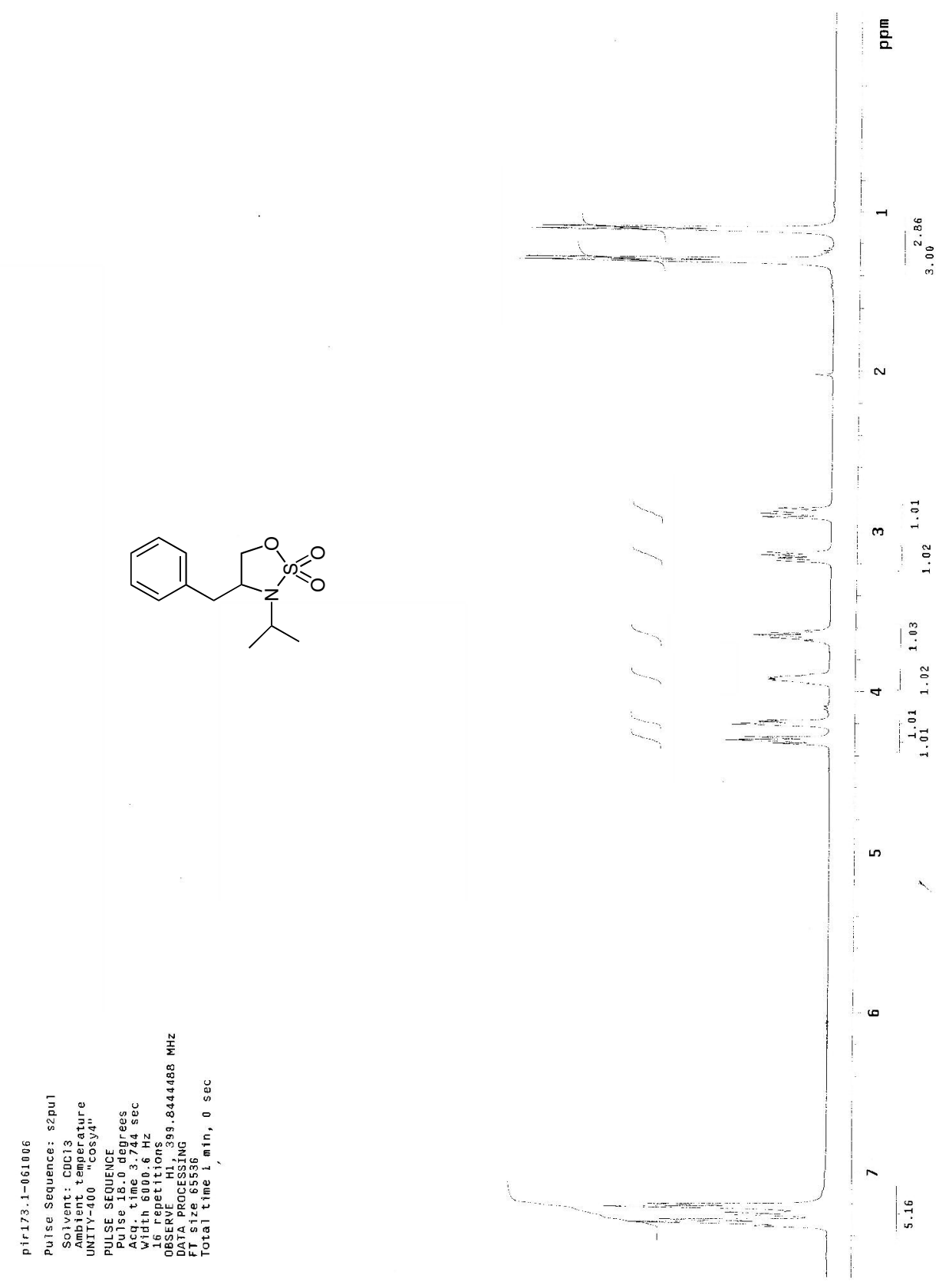


${ }^{13} \mathrm{C}$ NMR (4S)-3-isopropyl-4-benzyl-1,2,3-oxothiazolidine $S, S$-dioxide (5b)

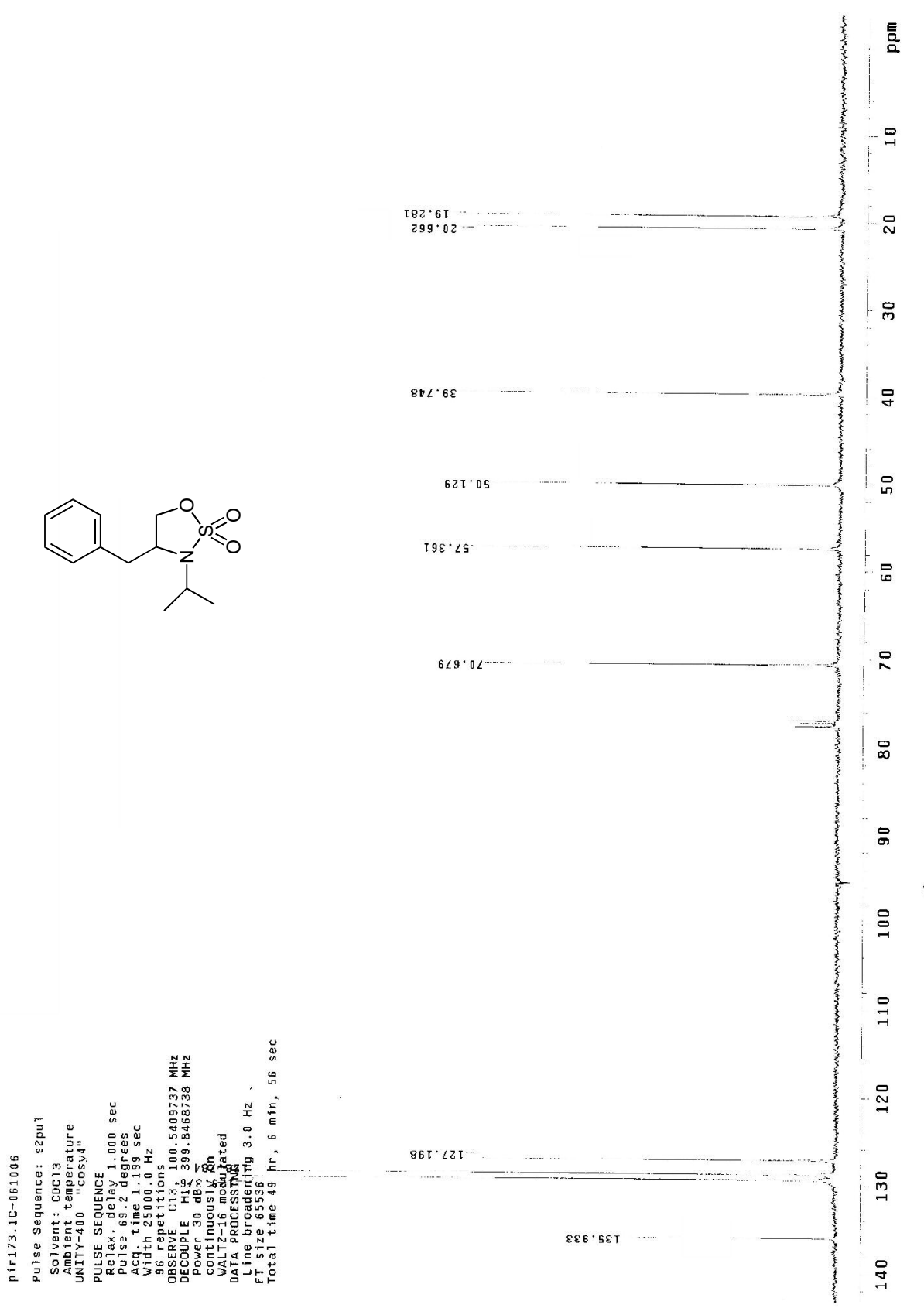


${ }^{1} \mathrm{H}$ NMR $(R)$-1-isopropylamino-1-phenyl-2-(diphenylphosphino)ethane (6c)

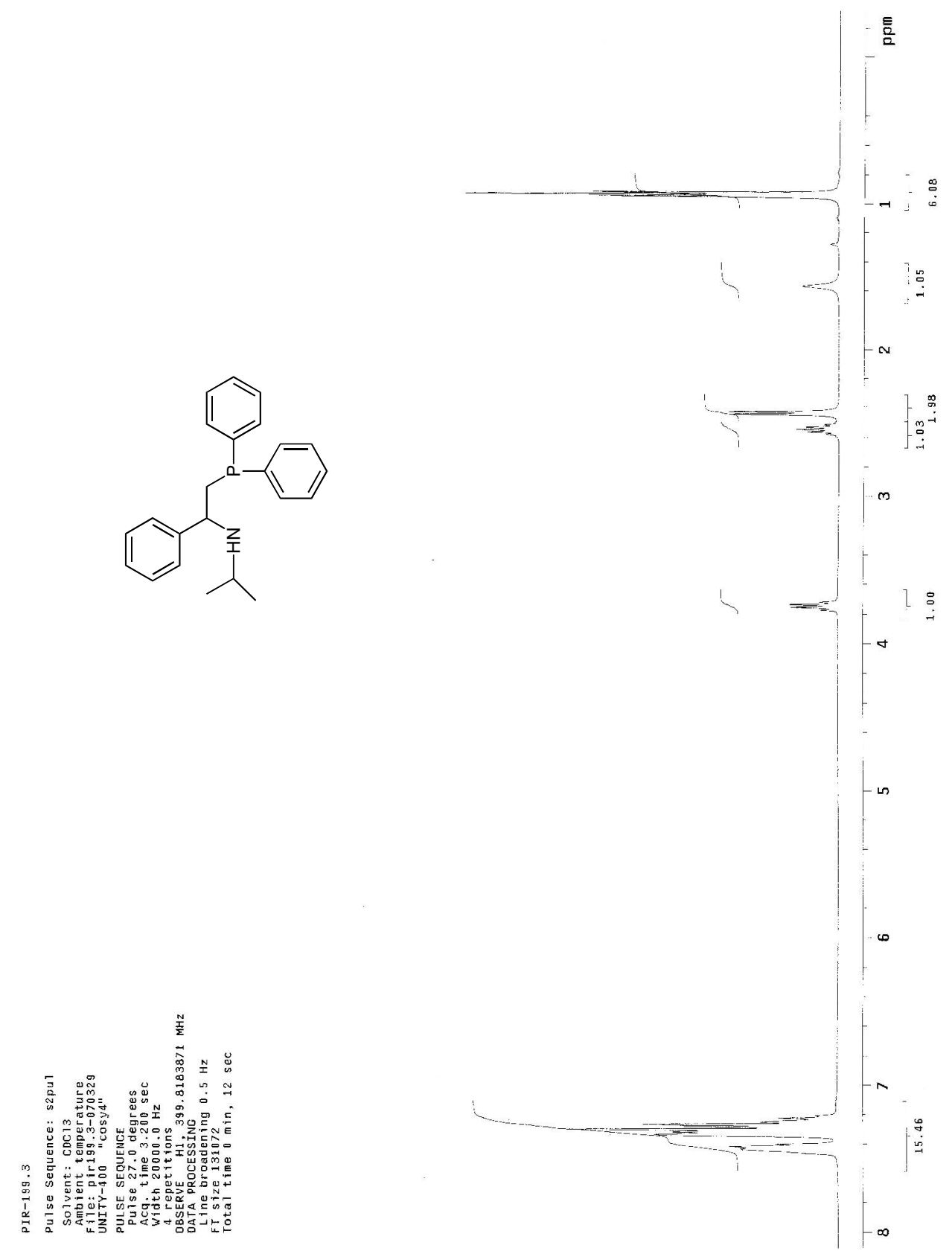


${ }^{13}$ C NMR (R)-1-isopropylamino-1-phenyl-2-(diphenylphosphino)ethane (6c)

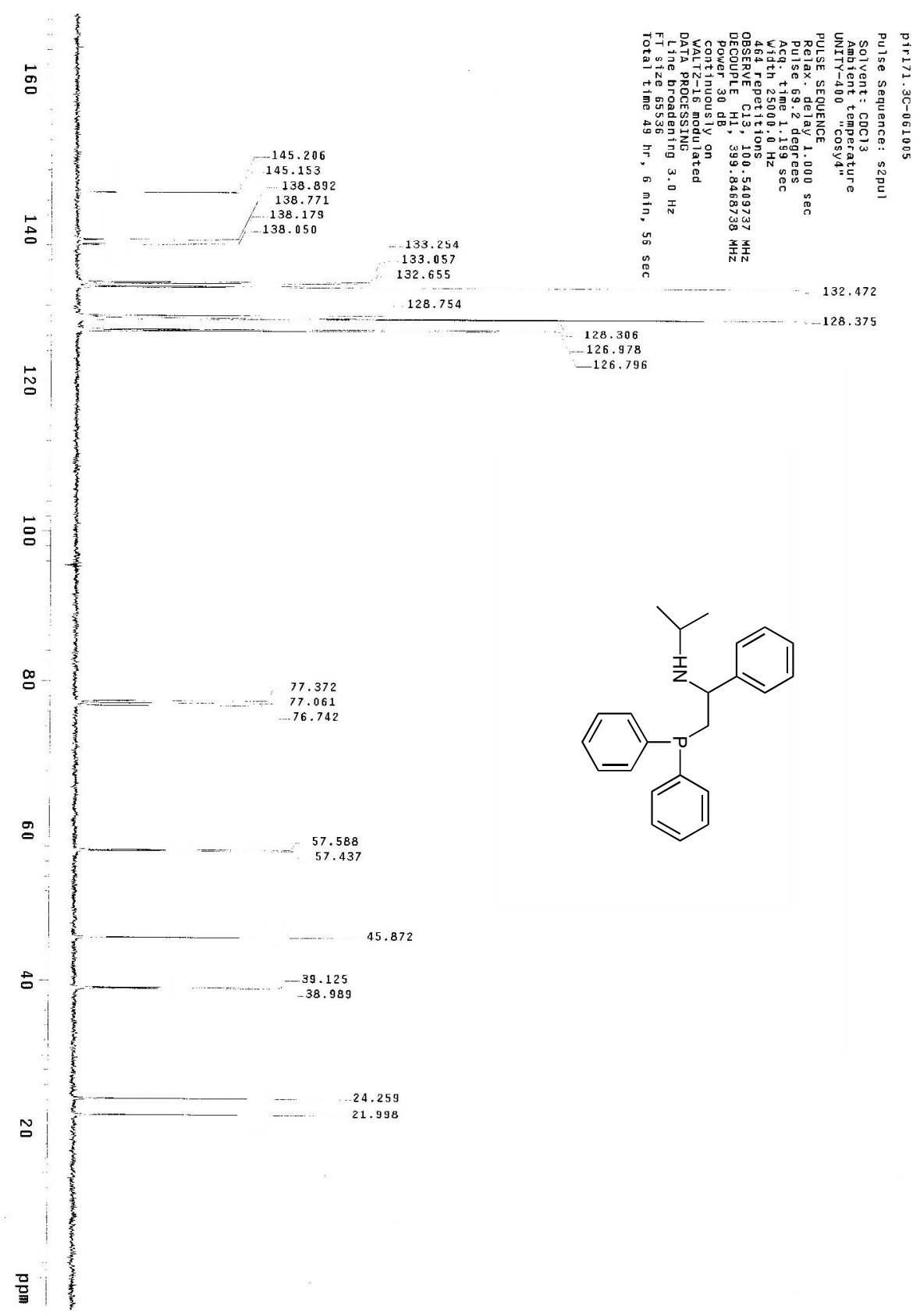


${ }^{1}$ H NMR (S)-2-isopropylamino-3-metyl-1-(diphenylphosphino)butane (6a)

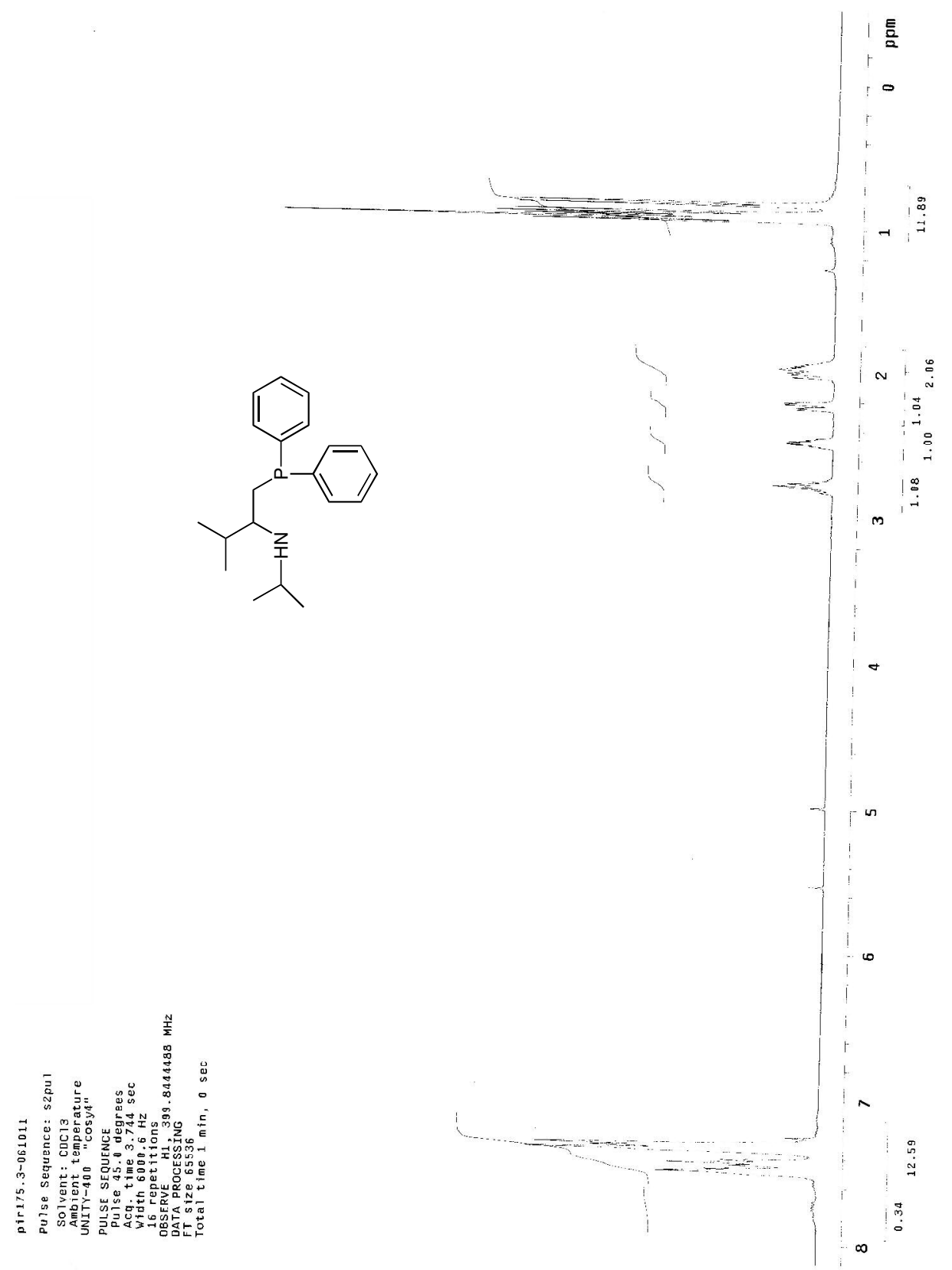


${ }^{13}$ C NMR (S)-2-isopropylamino-3-metyl-1-diphenylphosphinobutane (6a)

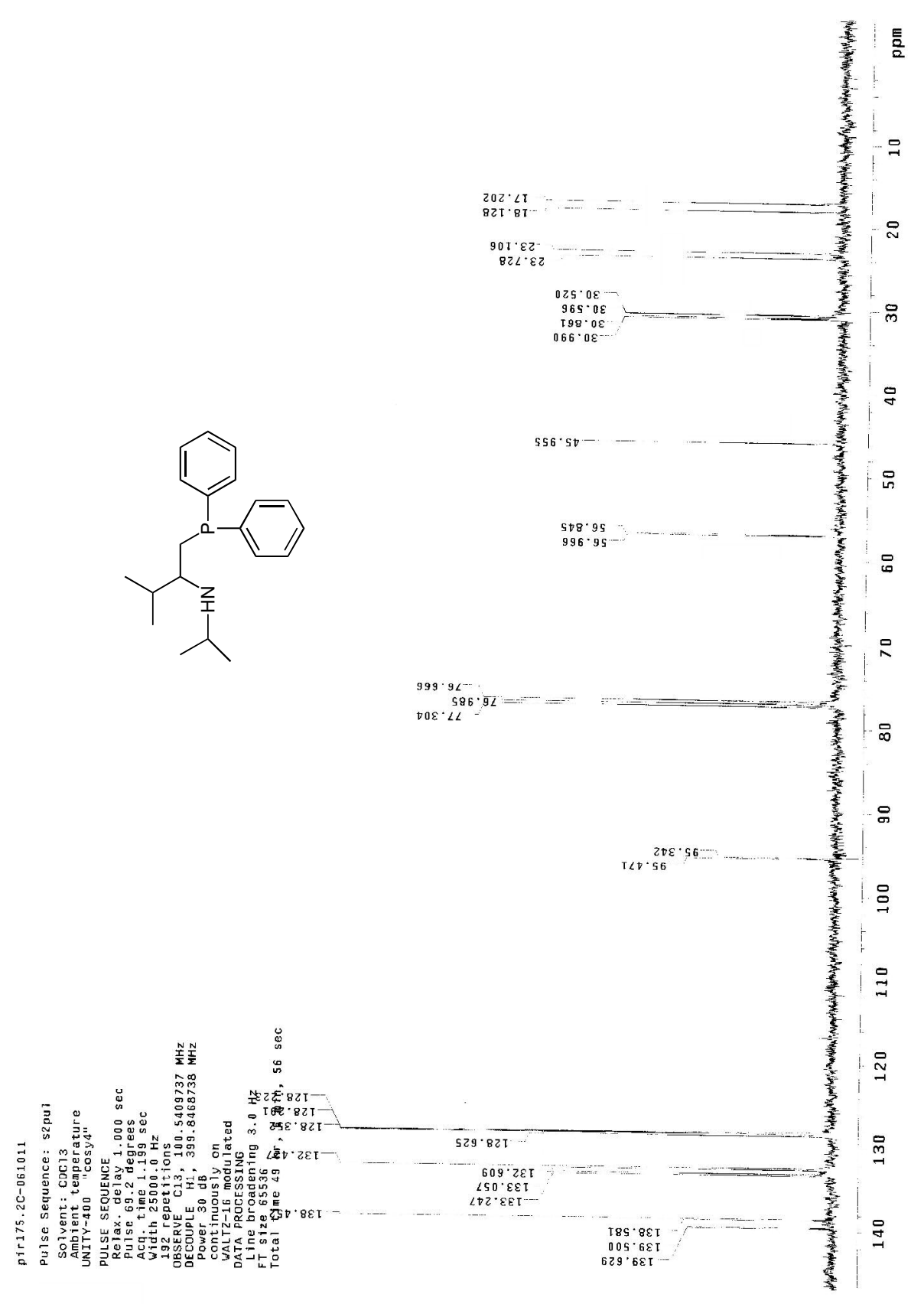


${ }^{1}$ H NMR (S)-2-isopropylamino-2-phenyl-1-(diphenylphosphino)ethane (6b)

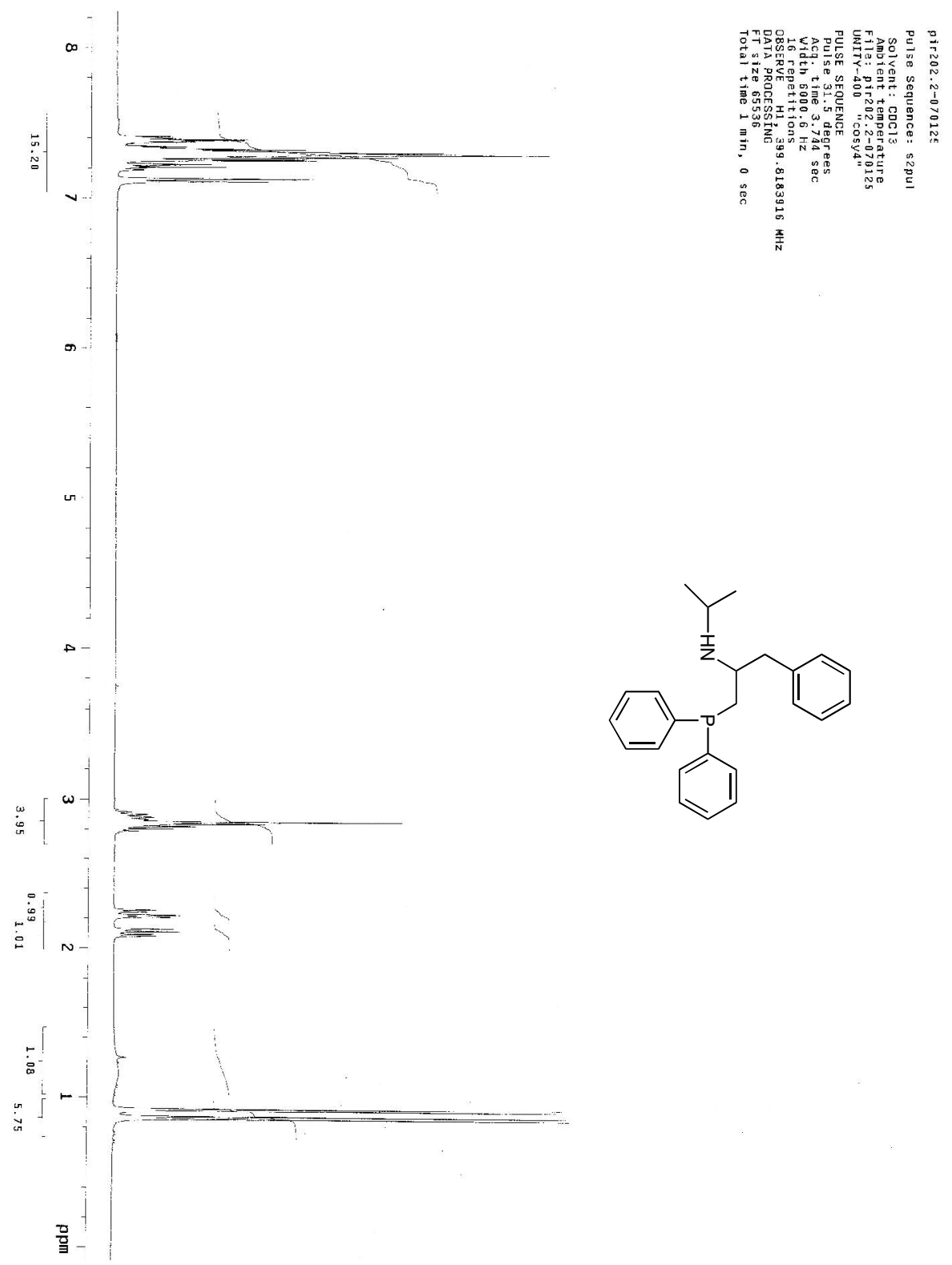


${ }^{13} \mathrm{C}$ NMR (S)-2-isopropylamino-2-phenyl-1-(diphenylphosphino)ethane (6b)

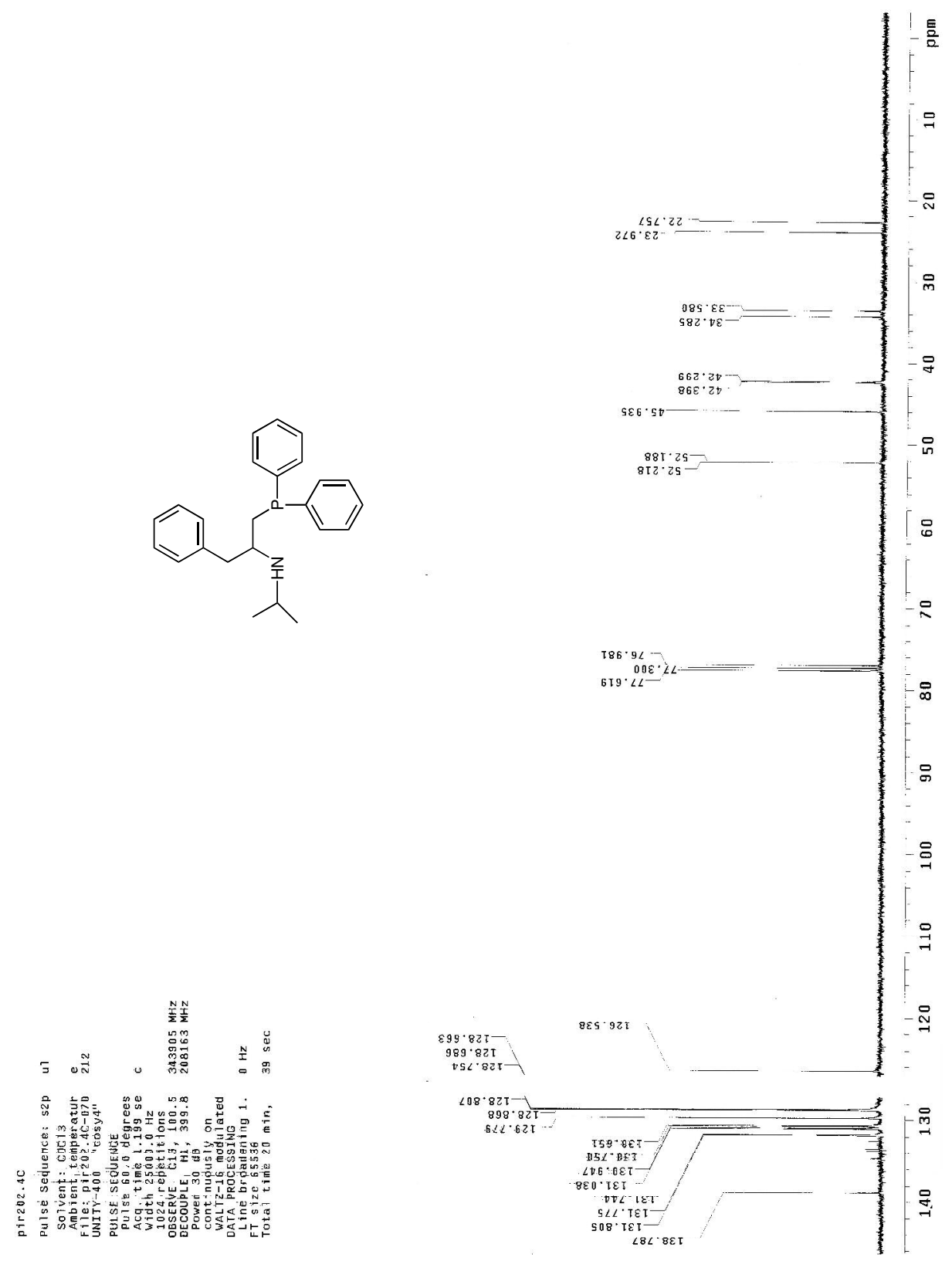

